\title{
9 Towards more natural interfaces
}

\author{
Jean-Marie Laborde \\ Laboratoire Leibniz-IMAG \\ Grenoble, France
}

\begin{abstract}
More natural interfaces are likely to be a main feature in the future design of Direct Manipulation Software, including Dynamic Geometry. This, however, will not be without problems in the actual design and in the tensions these new possibilities will bring due to constraints implied by the nature of the curriculum, specific content knowledge which is deemed to be important, and the expectations of teachers - the teachers' culture.
\end{abstract}

\section{Keywords}

Direct manipulation, geometry, human computer interface.

\section{A CONDENSED HISTORY OF RECENT MAN-MACHINE INTERFACE EVOLUTION}

At the end of the 70's, engineers at Rank Xerox conceived the idea of an interface for a computer essentially as a simulation of an office, actually proposing an electronic counterpart to it. The familiar environment of an office is presented as a desktop, on which we have folders containing files; some files can be put directly on the desktop, some files are open other are closed, showing only their titles; in addition we find on the desktop mail baskets, one for the outgoing mail, one other for the incoming mail, a printer could also be available. All this was present in the design of the Star Machine at the Rank Xerox Research Laboratories in Palo Alto. Only a trash was missing on this first attempt to create some sort of 'natural' interface. Just after Steve Jobs and his friends had received a shock in discovering and realising the potential of what was just happening at the Xerox Park, engineers at Apple introduced the trash, an easy way to destroy and possibly recover any file the user wanted to get ride of. Almost 25 years later, Windows 97 also finally brings a trash to the PC user community. Actually, Microsoft products have some look of modernity, its icon shows a recycling trash instead of the outdated waste trash. 
Direct Manipulation (DM): some difficulties

During the past twenty years not too much has been accomplished in comparison with the giant step forward accomplished at Rank, with the introduction of a computer interface based on an electronic counterpart of the physical world. Because it was now possible to act on an internal object through its graphic representation-looking almost as if it was real-the concept of direct manipulation (Schneiderman, 1987) was introduced to characterise such environments where the user acts on internal objects through some graphical representation produced for the user by the interface.

Despite its incredible potential, the general ideas of direct manipulation, enhanced through direct engagement, has not affected too much the majority of application software that people continue to produce and to use, especially in the educational software domain.

Possible reasons for this situation are:

- the difficulty in finding appropriate metaphors, relevant to the cultural background of the user as well as to the specific content of the knowledge domain covered by the application;

- the general difficulties in programming at a fine level of granularity the interaction at the level of the main input device (mouse-keyboard);

- the temptation of applying the concept in software design of 'divide and conquer' and to de-limit relatively separate sub-domains of activities, introducing an increasing amount of modal type interaction;

- the development of system architecture like windows, which has been designed by people strongly influenced by their DOS type culture and the way they conceive and use computers (an example here is way the mouse in the Windows Environment is put on the top of the architecture and not really part of the whole design of the system).

\section{SOME EXAMPLES IN MATHEMATICS (GEOMETRY)}

One can think about a geometrical figure as a set of objects related by some properties 'expressing' the figure itself. In a book a figure is commonly represented by a drawing in addition to text describing those relations as wanted by the designer of the figure. For instance a figure could represent two points which are symmetric with respect to some line (see figure 1). Normally, no remarkable property in the drawing can be found which would not be a 'logical' geometrical consequence of the properties as expressed by the designer. This means that the drawing is supposed somehow to be a model of the figure in its most general position (this is true up to some properties an expert would consider as irrelevant, e.g., the fact that a figure involving a triangle will often be presented as a triangle with an horizontal base). In the case above, suppose two points are 
labelled $A, B$ and the line $D$. $A$ can be viewed as the symmetric image of $B$ in the axial symmetry around $D$, as well $B$ can be viewed as the image of $A$.

Figure 1 A geometrical figure representing 2 symmetric points with respect to a line.

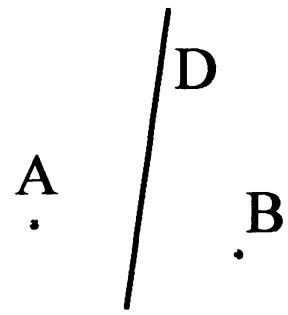

\section{Dynamic Geometry Software}

Dynamic Geometry Software, or DGS, refers to computer-based environments which appeared some years ago to help discover the properties of a figure through its behaviour when the user 'drags' some of its components with the mouse, usually the points the user has first introduced when creating the figure by a sequence of calls to primitives.

Taking the example of Cabri (Laborde, 1995), a figure constructed by a student in the above case could be one of the following:

- A point $A$ and a line $D$ have been created somewhere on the working sheet, not sharing any explicit relationship. Then the user created, through a call to the item axial symmetry, the image of $A$, namely $B$, around the axis $D$. It is possible to drag $A$ or $D$, but $B$ cannot be dragged despite the fact that $A$ is equally treated in the syntaxic description of the figure. At any time the Cabri-construction gives a drawing which can be viewed as a static drawing of the geometrical figure, but dynamically one would prefer a solution where $A$ or $B$ could each be dragged on their own.

- Instead of calling the primitive axial symmetry, another student might have constructed the perpendicular line from $A$ to $D$ and then considered the other intersection of this line with the circle centred at the foot of the perpendicular line, passing through $A$. In respect to the above consideration the behaviour of dragging this construction would be exactly the same.

- Another possibility would be to start from two points $A$ and $B$ and construct the perpendicular bisector of $A B$. This time $A$ and $B$ would be equal according to their drag capabilities, but as in the preceding case now the line $D$ would not behave any with full generality or freedom.

Using Geometer's Sketchpad (GSP: Jackiw, 1995) we will take advantage of the mirror feature producing the mirrored image of a point with respect to a line that we have just marked as 'current mirror'. The behaviour here is more general than for Cabri because it is possible to drag $A$ or $B$ apparently in an entire symmetric way. But if one drags the line $D, A$ takes over as a more fundamental object than $B$ and remains immobile.

In GSP, it appears that it is not easy to obtain the symmetric image of a point by using the construction of a perpendicular line and a circle, such that in all 
74 Information and communications technologies in school mathematics

cases the constructed point $B$ behaves as the symmetric image of $A$. In contrast, even if there is no perpendicular bisector primitive, we could construct a perpendicular bisector from two points-first constructing the midpoint of the line-segment joining them and a perpendicular line through the midpoint. The resulting construction behaves essentially the same way as in the case of Cabri.

\section{SOME IMPLICATIONS FROM AN EDUCATIONAL POINT OF VIEW}

As shown in the previous section, figures obtained in DGS corresponding to the same geometrical representation can behave differently, according to the way they have been constructed or according to the software program used in the construction. It is probably important to draw teachers' attention to this aspect of dynamic geometry. Actually, dynamic geometry (Euclidean, analytical or whatever else) as manifested in DGSs, appears relatively clearly as something different from geometry. According to experience gained in looking at different kind of users using DGS (e.g., teachers, students, mathematicians), one can say that there is no one preference. For instance, very often the relative freedom preserved in GSP about dragging (the essence of dynamic geometry) is appreciated by teachers, especially in the US. In France, when this kind of behaviour has been deliberately introduced with Cabri II the reaction was strongly negative among those users (teachers) who discovered it. These users were claiming that the software was no longer keeping a 'logical dependence' among the different objects constructed.

This indicates the need for further research into the conceptions or misconceptions that can be created due to some inherent characteristics of dynamic geometry, and also into some of the design choices made at the software design level.

Actually, if we think about a DGS as an environment to help model some situation, e.g., in mathematics or physics, we could require a DGS to behave in a considerably more 'permissive' way. Consider, for example, a mechanical device in which we have a wheel tangent to two guides, represented by two concurrent lines. As a physicist one would be interested in producing a figure like that given in figure 2, in the fastest and easiest possible way. Using current Cabri or GSP we would have to consider a point on a line, then a perpendicular line, to produce finally, after a whole set of intermediate constructions, the circle representing the wheel. 


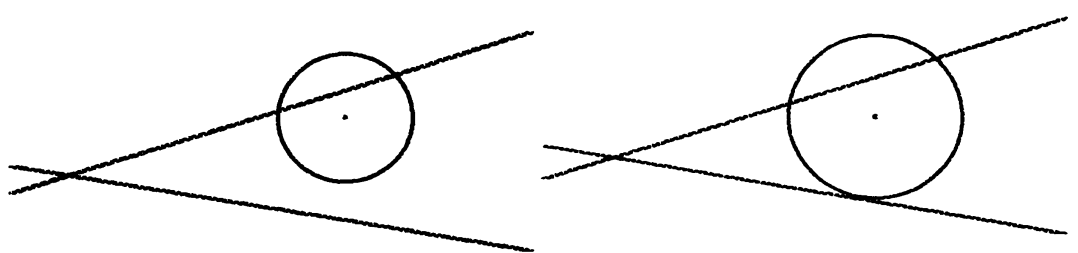

Step 1 Create a circle.

Step 2 Make it tangent to one of the two lines.

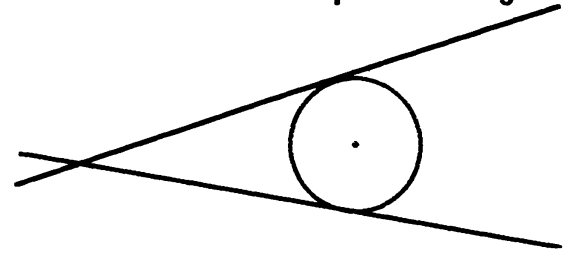

Step 3 Making it tangent to the other line.

Figure 2 Obtaining a circle tangent to two lines.

In my opinion it would be highly desirable to have an interface which would enable the user to obtain the same figure through the creation of a circle, then approaching its periphery from one of the lines making it 'visibly' tangent to the line, and finally doing the same for the other line. From a mathematical education perspective, the possibility of achieving the desired outcome through first realising them visually would help the user to 'discover' what are the extra properties shown by the figure in its final stage. This in turn could help the user in 'constructing' the expected circle in a more traditional way (using basic primitives). For instance it would be possible to check that the locus of the centre of the circle is a line, actually the angle bisector of the two lines. The latter property seems to be obvious to a mathematics teacher (an expert) but this cannot at all be assumed in the case of a beginner (a student).

In the same spirit one could also think about allowing the user to constrain a point to be part of an object, simply by dragging it onto the object (if possible), and in the very same way to free a point from an object simply by dragging it with the mouse with an emphasis somehow in a direction roughly normal to the object or to its boundary.

\section{MORE NATURAL INTERFACES: THE TENSION}

Considering that students are normally coping with some well-defined set of construction problems (see, for example, Hoelzl, 1995), it is likely that some people will refrain from accepting an interface which provides the generality or freedom implied in the example given above. An initial reaction, at least for 
mathematics teachers, may be that such flexibility would no longer require the use of the underlying 'formal' geometric structures or relationships in the constructions. In which case they may well be overlooking the potential the new features might have for providing insights as to how subsequent construction(s) might be developed in a more precise manner using the more formal aspects of the mathematics (as indicated in the discussion of the example), if in fact, this is the desired final purpose of the DGS activity. Nevertheless, if we really do have Direct Manipulation and Direct Engagement in mind, one can only think that this flexibility in interface is about to be the future.

\section{REFERENCES}

Hoelzl, R. (1995). Between Drawing and Figure. In R. Sutherland and J. Mason (eds.) Exploiting Mental Imagery with Computers in Mathematics Education. NATO ASI Series, Berlin. Heidelberg: Springer-Verlag, 117-124.

Jackiw, N. (1995). Geometer's Sketchpad (Version 3). Berkeley, California: Key Curriculum Press.

Laborde, J-M. (1995). Cabri-Geometry II, Franck Bellemain designers, TI Dallas, Texas.

Scneiderman, B (1987). Designing the User Interface, Strategies for Effective Human-Computer Interaction. Reading (Massachusetts): Addison-Wesley.

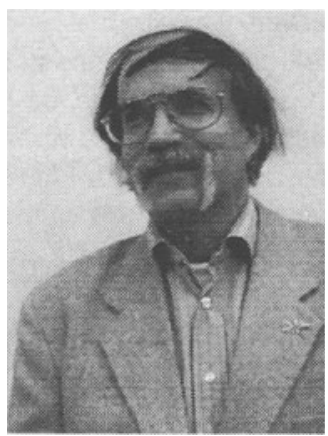

Jean-Marie Laborde graduated in mathematics from the École Normale Supérieure in Paris and started his research work specialising in the use of geometric methods for the study of graphs, especially hypercubes and also automatic theorem proving. With others he started the Cabri project in 1981, initially as an environment for graph theory. In 1987 a number of students and young researchers joined the project to start Cabri-géomètre, a software program available for computers and now incorporated in a new type of calculator, the Texas Instrument TI-92. 